\title{
Moving Away from the Tip of the Pyramid: Screening and Brief Intervention for Risky Alcohol and Opioid Use in Underserved Patients
}

\author{
Kamilla L. Venner, PhD, Victoria Sánchez, DrPH, Jacqueline Garcia, MPH, \\ Robert L.Williams, MD, MPH, and Andrew L. Sussman, PhD, MCRP
}

Purpose: Rates of risky substance use and substance use disorders are high in primary-care practices, yet the adoption of universal screening and brief intervention (SBI) has been slow and uneven. This study aimed to describe SBI-related attitudes, practices, and perspectives regarding practice change among medical providers in a minority-majority state.

Methods: We conducted a cross-sectional, on-line survey of a practice-based research network of medical providers serving predominantly Hispanic/Latinx and Native American patients in rural and urban settings. The main variables were clinician 1) perspectives on the need to address substance use problems in primary care, 2) current screening and intervention practices, and 3) satisfaction with and willingness to make changes to their practices.

Results: Although providers endorsed alcohol and opiate misuse to be significant problems in their practices, only $25 \%$ conducted universal screening. Providers reported focusing most of their screening efforts on those with substance use dependence. In general, providers rated importance of and ability to make practice changes moderately high. There was high interest in practice coordination with the community followed by interest in a collaborative care approach.

Conclusions: Providers mainly focus efforts on the relatively few patients at the tip of the pyramid (substance use dependence) rather than on the majority of patients who comprise the middle of the pyramid (risky substance use). Practice change strategies are needed to increase universal screening with a focus on risky substance use, particularly in practices serving racial/ethnic communities. (J Am Board Fam Med 2018;31:243-251.)

Keywords: Cross-Sectional Studies, Hispanic Americans, Native Americans, Primary Health Care, Substance Abuse

The integration of screening and brief interventions (SBIs) for patients with unhealthy alcohol and drug use in primary care continues to represent a significant challenge for clinicians in their efforts to address an expanding list of preventable health

This article was externally peer reviewed.

Submitted 20 March 2017; revised 7 November 2017; accepted 13 November 2017.

From the Department of Psychology and Center on Alcoholism Substance Abuse, and Addiction (KLV), College of Population Health (VS), Department of Family and Community Medicine (JG, RW, ALS), University of New Mexico, Albuquerque, NM.

Funding: Research reported in this publication was supported by the National Institute on Minority Health and Health Disparities of the National Institutes of Health under Award 1P20MD004811-01. The content is solely the responsibility of the authors and does not necessarily represent the official views of the National Institutes of Health.

Conflict of interest: none declared. problems. ${ }^{1}$ Strong evidence supports universal screening for alcohol use while federal agencies encourage screening for drug use, especially in the context of illicit drug and prescription drug misuse. ${ }^{2-5}$ Despite these recommendations and awareness regarding the scope of the problem, research has consistently documented missed opportunities for detecting these problems. ${ }^{6}$

Efforts to assist primary care providers have addressed some, though certainly not all, of the existing barriers, such as time limits and lack of

Prior presentation: Oral presentation at the New Mexico Public Health Association Annual Conference, Albuquerque, NM, March 31, 2015.

Corresponding author: Victoria Sánchez, Dr PH, 1 University of New Mexico, MSC 09 5070, Albuquerque, NM 87131 (E-mail: ViSanchez@salud.unm.edu). 
financial resources. ${ }^{7}$ Lengthy screening questionnaires have been reduced to a single question, motivational interviewing techniques have demonstrated effectiveness and can be deployed during the brief encounter, and patient-centered medical home models provide guidance about the integration of other practice staff in these efforts. ${ }^{8-11} \mathrm{Al}-$ though these resource-focused solutions are helpful, they may not adequately acknowledge another important dimension of the SBI dissemination problem: the "prevention paradox." 12 The prevention paradox refers to research confirming that the majority of overall adverse health effects (eg, increased mortality, injury, and life problems) ${ }^{13}$, are attributable to a larger group (59\%) of drinkers that fall into the low- or high-risk alcohol use categories compared with the $13.9 \%$ that meet past-year alcohol use disorder (AUD) criteria. ${ }^{14-15}$ These percentages of drinking practices have been commonly represented within a pyramid (see Figure 1). In practice, there may be a stronger emphasis on identifying and treating patients at the "tip of the pyramid" (eg, patients who meet criteria for AUD) while the guidelines emphasize that stronger evidence exists for SBI efforts for those at earlier stages. ${ }^{8}$ This potential misalignment of SBI counseling resources may help explain low rates of clinician self efficacy in managing patients with substance use disorders. ${ }^{1}$

In New Mexico, a geographically expansive state with both current and historically highest death rates from alcohol and drug overdose, the need to expand SBI is particularly urgent. ${ }^{16-17} \mathrm{We}$ conducted a cross-sectional survey on SBI attitudes and practices among primary care clinicians in Research

Figure 1. A pictoral representation of percentage of the US population drinking at various levels of risk for developing alcohol use disorder. Data are from Dawson et $\mathrm{al}^{14}$ and Grant et $\mathrm{al}^{15}$.

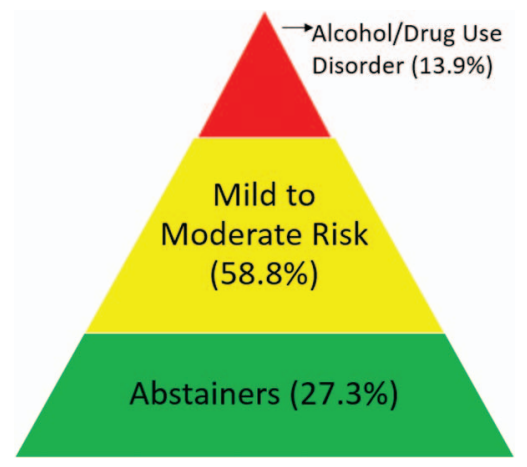

Involving Outpatient Settings Network (RIOS Net), a practice-based research network in New Mexico, serving low-income, predominantly Hispanic/Latinx and Native American communities. The aims of the survey were to characterize primary care clinician views about management of risky alcohol and drug use and explore their practices and perceived barriers to enhanced SBI practices in medically underserved communities. We compared views on alcohol versus opiate SBI. This survey represents the first step within a multi-stage research project toward identifying potential strategies to inform practice-based interventions aimed at expanding SBI to those patients in the middle of the pyramid.

\section{Methods}

\section{Study Design and Setting}

We conducted a cross-sectional survey of primary care providers in RIOS Net, a practice-based research network in New Mexico. We excluded pediatrician members of the network given our focus on the adult patient population. The study was approved by the University of New Mexico Human Research Protections Office.

\section{Measures}

The research team developed a survey to assess clinician approaches and attitudes to SBI for alcohol and opiate misuse in primary care settings in medically underserved communities (see Appendix 1 for the full survey). We reviewed substance abuse literature and consulted with clinicians who specialize in alcohol and drug use health services research to create the survey. We reviewed validated instruments to guide development of our final survey (eg, Organizational Readiness for Change ${ }^{18}$, Assessment of Chronic Illness Care ${ }^{18}$ ). A total of 6 RIOS Net Executive Board members and Department Family Medicine Residents piloted the survey. Survey items included scaled (visual analog 1 to 100 , eg, $1=$ not at all helpful or strongly disagree and $100=$ significant or strongly agree), multiple choice, and 1 ranking of priorities question. Items focused on clinician ratings of the need to address substance use problems in primary care, current screening and intervention practices and satisfaction with and willingness to make changes to their practices. 


\section{Data Collection}

We administered the provider survey electronically through Research Electronic Data Capture $\left(\right.$ REDCap $^{19}$ ), a web-based electronic data capture program. Nonresponders were sent additional solicitations at 7-to-10-day intervals. After 5 email solicitations, article surveys were mailed to nonresponders. This was followed by another mailing 2 weeks later. As an incentive, all respondents were entered into a pool and were eligible to win gift cards. The survey took approximately 15 minutes to complete and respondents earned 1.5 units of continuing medical education credits. At the conclusion of the survey, we included links (references on article-based questionnaires) to educational activities and clinical resources on SBI for alcohol and drug use. We exported the anonymous data for statistical and qualitative analysis.

\section{Data Analysis}

Responses from clinicians who used the web-based questionnaire were stored in the REDCap database. Article-based responses were double-entered by research staff into Microsoft Excel. Analyses of all data were done using Stata software. To report results of the survey, we calculated the mean and standard deviation of each continuous survey response. Further, we explored differences between continuous responses for alcohol versus opiates using paired $t$-tests. Categorical responses were summarized using frequencies. To explore differences between responses for alcohol versus opiates, the StuartMaxwell test, a generalization of McNemar's test for multiple categories, was used. For post-hoc analyses, we conducted ANOVAs to explore differences on survey responses based on years of practice. All statistical significance tests were 2-sided and conducted at the 0.05 significance level.

\section{Results \\ Participants}

The sampling frame consisted of 126 eligible clinician members of RIOS Net. The survey response rate was $55 \%(\mathrm{~N}=69 ; 45 \%$ women). The sample is described in Table 1. On average, respondents were 51.7 years of age ( $\mathrm{SD}=9.2$ years; range, 32 to 69 years) with 19.8 years of experience as a medical provider ( $\mathrm{SD}=10.0$ years, range, 1 to 40 years). The majority of respondents were family physicians
(68\%) and were distributed across University of New Mexico (32\%), Community Health Center (29\%), and Indian Health Service (21\%) outpatient primary care settings.

Overall, in line with our research aims and emergent findings, these results are organized in 4 categories: 1) perception of the problem and level of prioritization, 2) reported SBI approaches, 3) considerations for practice changes, and 4) posthoc exploratory analyses to examine variability in responses. Within each of these main categories,

Table 1. Summary of Health Professionals' Characteristics Expressed as Absolute Numbers and Percentage

\begin{tabular}{|c|c|c|}
\hline Characteristic & $(\mathrm{N}=69)$ & Percentage \\
\hline \multicolumn{3}{|l|}{ Practice specialty } \\
\hline Family physician & 47 & 68.12 \\
\hline Internist & 11 & 15.94 \\
\hline Physician assistant & 5 & 7.24 \\
\hline Nurse practitioner & 4 & 5.80 \\
\hline Other & 2 & 2.90 \\
\hline \multicolumn{3}{|l|}{ Primary clinical setting } \\
\hline University of New Mexico & 22 & 31.88 \\
\hline Community Health Clinic & 20 & 28.99 \\
\hline Indian Health Services & 15 & 21.74 \\
\hline Other & 6 & 8.70 \\
\hline Private practice & 5 & 7.24 \\
\hline Missing & 1 & 1.45 \\
\hline \multicolumn{3}{|l|}{ Experience (years) } \\
\hline 1 to 5 & 7 & 10.14 \\
\hline 6 to 10 & 8 & 11.60 \\
\hline 11 to 15 & 10 & 14.49 \\
\hline 16 to 20 & 8 & 11.60 \\
\hline 21 to 25 & 12 & 17.39 \\
\hline More than 25 & 23 & 33.33 \\
\hline Missing & 1 & 1.45 \\
\hline \multicolumn{3}{|l|}{$\begin{array}{l}\text { Total No. of staff in location } \\
\text { where you see the most } \\
\text { patients }\end{array}$} \\
\hline 1 to 5 & 11 & 15.94 \\
\hline 6 to 11 & 13 & 18.84 \\
\hline 11 or more & 42 & 60.87 \\
\hline Missing & 3 & 4.35 \\
\hline \multicolumn{3}{|l|}{ Gender } \\
\hline Male & 38 & 55.07 \\
\hline Female & 31 & 44.93 \\
\hline \multicolumn{3}{|l|}{ Age range (years) } \\
\hline 30 to 39 & 11 & 15.94 \\
\hline 40 to 49 & 16 & 23.19 \\
\hline 50 to 59 & 26 & 37.68 \\
\hline 60 to 69 & 16 & 23.19 \\
\hline
\end{tabular}


we present findings for addressing risky alcohol and opiate use followed by commonalities and differences in addressing these 2 substances. Table 2 presents survey item results.

\section{Perception of Problem and Level of Prioritization}

While not reaching statistical significance, we observed a trend toward providers reporting that opiate misuse was more of a problem in their patient population than was alcohol misuse $(P=.054)$. Respondents more strongly agreed that addressing opiate use was a priority than addressing alcohol. This higher rating for opiates may be attributable to the significant escalation in prescription pain medication misuse and overdose death rate in New Mexico and the nation. Providers also rated the degree to which their clinic leadership prioritized these topics at the same level, with opiate use once again higher than for risky alcohol use.

\section{Reported SBI Approaches}

We asked a series of questions assessing the circumstances that lead to screening. Providers reported relying mostly on their clinical impressions based on individual patient characteristics to detect substance use problems in contrast with a universal screening system. They reported this case finding approach at higher levels for patients with opiate problems (65\%) compared with alcohol problems (50\%). However, when looking at clinician rates of screening for opiate misuse at all patient visits $(15 \%)$ and for well visits (13\%), the rates almost doubled for alcohol screenings (26\% and $22 \%$, respectively). Only about a quarter of respondents indicated that they screened patients at every visit (eg, universal screening) for alcohol and this rate was lower for opiates.

When asked about who initiates screening in the practice, clinicians reported doing so more often than nurses/medical assistants and/or having patients complete a screening questionnaire. Clinician-led screening was reported at a higher level for opiates $(77 \%)$ than for alcohol (62\%). Congruently, there was moderate agreement that screening was the clinician's responsibility. Perceptions of clinic staff investment in addressing alcohol and opiate problems were rated similarly at the midpoint of the range. Lastly, consistent with our interestin understanding how clinicians direct screening efforts along the pyramid, we asked respondents to rank priorities for alcohol screening. Providers ranked "people meeting criteria for alcohol use disorder" as the highest priority with the second highest rating for "patients with mild/moderate alcohol use" and lastly "patients with no current alcohol use problems; I focus on prevention" (see Table 3).

Most clinicians reported that they provided a mix of substance use interventions within the practice combined with referring to community resources at equal levels for both alcohol and opiate problems. Clinicians reported similar levels of awareness of community resources for these patients. About half of participants perceived that patients felt comfortable seeking treatment for alcohol or opiate use problems.

\section{Considerations for practice changes}

Only about half of clinicians felt strongly or somewhat satisfied with how their practice addressed patients with opiate use problems; slightly more were satisfied with their practice's management of alcohol use problems. Considering this lack of satisfaction, almost three quarters of these clinicians felt that making changes in practice approaches to these problems was important, and about the same percentage felt confident their practices could make the improvements needed (see Table 2). When asked about different strategies for improved care that might be considered, the highest level of interest was in increased coordination with community resources and learning collaborative approaches.

\section{Post-hoc Analysis of Data Variation}

We observed large variability across the visual analog questions (0 to 100), so we conducted post-hoc ANOVAs to examine potential clinician characteristics, including years of experience, for associations with screening attitudes and behaviors. ${ }^{20} \mathrm{We}$ divided the groups into 3 categories based on years of practice: $\leq 10$ years $\left(\mathrm{Mdn}_{1}\right), 11$ to 19 years $\left(\mathrm{Mdn}_{2}\right)$, and $\geq 20$ years $\left(\mathrm{Mdn}_{3}\right)$. Four questions yielded significant differences in ratings based on years of practice : 1) practice staff felt invested in patients with alcohol problems $\left(\operatorname{Mdn}_{1}=74, \operatorname{Mdn}_{2}=56\right.$, $\left.\mathrm{Mdn}_{3}=48 ; P=.011\right)$, 2) clinic leadership prioritized alcohol problems $\left(\mathrm{Mdn}_{1}=80, \mathrm{Mdn}_{2}=54\right.$, $\left.\mathrm{Mdn}_{3}=50 ; P=.032\right), 3$ ) increasing practice efforts to address alcohol problems was important $\left(\mathrm{Mdn}_{1}=88, \mathrm{Mdn}_{2}=66.5, \mathrm{Mdn}_{3}=71 ; \mathrm{P}=.027\right)$, and 4) noted interest in a collaborative approach care model $\left(\operatorname{Mdn}_{1}=88, \operatorname{Mdn}_{2}=84, \operatorname{Mdn}_{3}=73\right.$; $P=.035)$. Those with fewest years of practice rated 
Table 2. Medical Provider Responses to Survey about Alcohol and Opiate Screening, Brief Intervention, and Referral to Treatment in Primary Care

Survey Question

Thinking about your practice and patients generally, please indicate the degree to which alcohol/opiate use is a problem (Q1, Q17)

Compared to other health issues, addressing alcohol/opiate use problems is a top priority for our practice (Q6, Q21)

The leadership in my practice considers addressing alcohol/opiate use problems a priority (Q9, Q24)

As a primary care clinician, it is my responsibility to address alcohol/opiate use problems in my patients rather than referring them to others in the community (e.g. specialty clinics) (Q7, Q22)

Practice staff (other than physicians and mid-level practitioners) feel invested in helping patients with alcohol/opiate use problems (Q8, Q23)

Which of the following statements best describes the current situation in your practice for screening patients for alcohol/opiate use problems? (Q2, Q18)

Screening based on individual patient characteristics

Screen at all patient visits

Screen at well patient visits

Never screen

When patients are screened for alcohol/opiate use problems, which of the following statements best describes your approach? (Q3, Q19)

Clinician conducts screening

Nurse or medical assistant

Patient

In thinking about your practice as a whole, which of the following best describes your approach to treatment for patients with alcohol/opiate use problems? (Q5, Q20)

Mix of providing SUD treatment in practice and referring to outside resources

Only refer out to outside resources

Patients in my community feel comfortable seeking treatment for alcohol/opiate use problems at our clinic (Q11, Q26)

Are you satisfied with how your practice addresses patients with alcohol/opiate use problems? (Q15, Q30)

Strongly satisfied

Somewhat satisfied

Somewhat dissatisfied

Strongly dissatisfied

Increasing our efforts to intervene in patients' alcohol/opiate use problems is important to this practice (Q14, Q29)

I believe that my practice has the ability to make improvements in how we deliver care for patients with alcohol/opiate use problems (Q13, Q28)

If you feel that your practice could improve its care for patients with alcohol/opiate use problems, please indicate how helpful each of the following possible strategies might be:

Clinician training in, and structures to support use of, medications for alcohol/opiate use problems (e.g., naltrexone) (Q16a, Q31a)

Alcohol Mean (SD) Opiate Mean (SD) Significance Testing $(P)$

$65(22)$

$56(21)$

49 (25)

$\begin{array}{cc}50 \% & 65 \% \\ 26 \% & 15 \% \\ 22 \% & 13 \% \\ 1 \% & 7 \%\end{array}$

$63 \%$

$19 \%$

$16 \%$

$77 \%$

$10 \%$

$8 \%$

$\begin{array}{cc}55 \% & 57 \% \\ 37 \% & 25 \% \\ 52(24) & 48(27)\end{array}$

$\begin{array}{cc}9 \% & 12 \% \\ 49 \% & 36 \% \\ 35 \% & 39 \% \\ 7 \% & 13 \% \\ 69(22) & 74(21)\end{array}$

$66(22)$

68 (23) 


\begin{tabular}{|c|c|c|c|}
\hline Survey Question & Alcohol Mean (SD) & Opiate Mean (SD) & Significance Testing $(P)$ \\
\hline $\begin{array}{l}\text { A "collaborative care" approach (e.g., coordinated by a } \\
\text { nurse care manager) (Q16b, Q31b) }\end{array}$ & $75(23)$ & $76(24)$ & .654 \\
\hline $\begin{array}{l}\text { Training in behavior change approaches (e.g., } \\
\text { Motivational Interviewing) (Q16c, Q31c) }\end{array}$ & $68(20)$ & $64(25)$ & .023 \\
\hline $\begin{array}{l}\text { Increased practice coordination with other community } \\
\text { resources (e.g., treatment, social supports, etc.) (Q16d, } \\
\text { Q31d) }\end{array}$ & $82(14)$ & $78(19)$ & .055 \\
\hline $\begin{array}{l}\text { Effective instruments to help detect problem alcohol/ } \\
\text { opiate use (Q16e, Q31e) }\end{array}$ & $67(21)$ & $68(22)$ & .718 \\
\hline
\end{tabular}

Percentages may not add up to 100 due to rounding error. Numbers ranged from 1 to 100 unless a \% is noted. Significance at $P<$ .05 (bolded). "Q" refers to the question in the survey in Appendix 1.

SD, standard deviation; SUD, Substance Use Disorder.

these questions higher than those with more years of practice. For opiate-related questions, 1 significant difference: those with fewer years of practice noted interest in training on effective instruments to detect opiate use problems $\left(\mathrm{Mdn}_{1}=80, \mathrm{Mdn}_{2}=\right.$ 73.5, $\left.\mathrm{Mdn}_{3}=66.5 ; P=.025\right)$.

\section{Discussion}

We surveyed primary care providers' perspectives and reported practices regarding SBI for risky alcohol and opiate use in a rural Southwestern, minority-majority state. In general, primary care providers reported alcohol and opiate use to be problems in their practice and mostly reported similar management approaches with both problems. Despite United States Preventive Services Task Force (USPSTF) recommendations for universal screening for alcohol misuse ${ }^{5}$, only a quarter of providers reported universal screening for alcohol. Low rates of universal screening are consistent with previous findings that only one third of adults reported their primary care providers asked about alcohol and drug use. ${ }^{20}$ Although the USPSTF does not endorse universal screening recommendations for drug use, $15 \%$ of our participants reported doing so for opiates. This is substantially lower than a national survey of physicians, which reported $68 \%$ asked new patients about illicit drug use. ${ }^{21}$ Most providers reported both relying on their clinical judgment to identify alcohol and opiate problems and focusing their efforts on patients at the extreme end of the alcohol use spectrum (ie, those at the "tip of the pyramid").

While recognizing the important role that primary care providers play in addressing patients with severe substance use disorders ("tip of the pyramid"), there is a need to simultaneously refocus providers' efforts to patients in the middle of the pyramid in concordance with available evidence. Our findings reflect some of the known challenges associated with delivering SBI in primary care while also presenting a view of opportunities to make changes.

Collectively, our findings suggest that clinicians lead SBI efforts in their practice settings for select patients who they believe, based on clinical impressions, may be at a higher risk for a substance use disorder. At the same time, they endorsed survey items that may lead to enhanced attention to middle of the pyramid patients. Providers strongly endorsed collaborative care models and increased practice coordination with community resources as strategies to enhance SBI. Important for successful

Table 3. Medical Provider Rankings of How they Focus Their Screening Efforts

\begin{tabular}{lccr}
\hline Patient Category & \% Ranking 1st Priority & \% Ranking 2nd Priority & \% Ranking 3rd Priority \\
\hline Patients with dependent alcohol use & $\mathbf{5 6 . 9}$ & 21.9 & 20.6 \\
Patients with mild/moderate alcohol use & 33.9 & $\mathbf{5 9 . 4}$ & 6.4 \\
Patients with no current alcohol problems & 9.2 & 18.8 & $\mathbf{7 3 . 0}$ \\
\hline
\end{tabular}

Note: From Q 4 in the survey: We are interested in which patients you focus your alcohol screening efforts on. Please rank the following list ( 1 = highest priority; 3 = lowest priority) to show how you prioritize your efforts. The bolder numbers represent the highest percentage ranking at 1st, $2 \mathrm{nd}$, and $3 \mathrm{rd}$ for where clinicians focus their alcohol screening efforts. 
change, providers reported confidence in their ability to make practice-level changes. Clearly, these new models will be important in creating more comprehensive team-based changes aimed at broadening the scope of SBI to the middle levels of the pyramid. ${ }^{22}$

Our findings are consistent with emerging models of integrating primary care and behavioral health. Results from this survey suggest that efforts to increase coordinated care of this type are both desired and needed. Efforts should focus on both overcoming identified barriers as well as developing successful strategies for disseminating and implementing culturally appropriate SBIs. ${ }^{23-24}$ Strategies that may also be helpful to promote practice change include identifying practice "champions" of $\mathrm{SBI}^{25}$, ensuring training and dissemination of definitions of risky drinking; introducing evidence-based SBI approaches; updating practices on billing codes to cover SBI; and equipping providers with feasible SBI counseling techniques that fit the brief encounter. ${ }^{8,26-28}$

While the lack of evidence to support screening for other drugs is problematic, our survey findings indicate that these providers are actively managing these patients and desire training and practice changes to improve care delivery. There has been a call for more research on screening for risky drug use to guide providers to improve patient care. ${ }^{29}$ Even in the absence of evidence to support action in screening for drug use, there still may be compelling and ethical reasons for medical providers to consider screening for drug use such as 1) prescription drug interactions, 2) health concerns, and the 3) alarming increase in opiate overdose and mortality. ${ }^{29}$

\section{Cultural and Geographic Context}

These survey findings reflect the views and experience of providers with large percentages of Hispanic/Latinx and Native American patients in New Mexico. Racial/ethnic populations experience substantial substance misuse related health disparities ${ }^{17,30}$, underlining the compelling need to find effective and feasible approaches to this problem in primary care. Extending SBI access and moving away from the tip of the pyramid in culturally diverse settings will likely require adapting or expanding current "best practices" to increase culturally appropriate SBI practices and patient receptivity. For instance, one screening study in a hospital setting found greater reductions in drinking and drug use among Non-Hispanic White and African
American patients compared with Hispanic/Latino patients indicating potential differences across cultural groups. ${ }^{31}$ Future research should attend to the cultural context and various social determinants of health, such as poverty, discrimination, and neighborhood factors, to examine barriers and ways to facilitate culturally effective SBI.

\section{Limitations}

These findings on primary health care providers' perspectives on SBI and referral to treatment must be taken in the context of the limitations of the research. Although the response rate was acceptable, the sample size of providers was relatively small and based on respondents from a set of providers in underserved communities in New Mexico. These results may not generalize to other locations beyond the Southwest. However, clinician respondents were experienced (average, 20 years) and included statewide representation with patient population diversity. Finally, New Mexico consistently ranks high nationally in both prescription drug overdose and alcohol death rates. Therefore, developing a better understanding of clinician observations about SBI barriers and facilitators from these types of settings may offer greater potential to inform primary care practice change efforts.

\section{Conclusion}

Through this cross-sectional survey of SBI for alcohol and opiate misuse, we sampled clinicians in a practice-based research network primarily serving American Indian and Hispanic/Latinx populations and found low rates of universal screening practices. Most screening was targeted to specific individuals based on clinical judgment and these medical providers reported prioritizing screening patients with substance use dependence (the tip of the pyramid) rather than patients with risky substance use (the middle of the pyramid). One strategy that may help practices move to universal screening is to engage, as appropriate, other practice staff in coordinated screening models, while another may be through integration of behavioral health providers into primary care. Finally, given the alarming substance-related health disparities among racial/ethnic populations and other underserved groups ${ }^{16,17,30}$, future research is needed to examine cultural factors and social determinants of health that may influence the successful implemen- 
tation and impact of SBI among our rapidly diversifying nation.

We gratefully acknowledge Drs. Lauren Hund and Betty Skipper for their assistance with statistical analyses.

To see this article online, please go to: http://jabfm.org/content/ 31/2/243. full.

\section{References}

1. Elwy AR, Horton NJ, Saitz R. Physicians' attitudes toward unhealthy alcohol use and self-efficacy for screening and counseling as predictors of their counseling and primary care patients' drinking outcomes. Subst Abuse Treat Prev Policy 2013;8:17.

2. Saitz R, Palfai TP, Cheng DM, et al. Screening and brief intervention for drug use in primary care: The ASPIRE randomized clinical trial. JAMA 2014;312: 502-13.

3. Solberg LI, Maciosek MV, Edwards NM. Primary care intervention to reduce alcohol misuse ranking its health impact and cost effectiveness. Am J Prev Med 2008;34:143-52.

4. National Institute on Drug Abuse. Screening for drug use in general medical settings: Resource guide. Available from: https://www.drugabuse.gov/sites/ default/files/resource_guide.pdf. Accessed April 20, 2016.

5. Moyer VA, Preventive Services Task Force. Screening and behavioral counseling interventions in primary care to reduce alcohol misuse: U.S. Preventive Services Task Force recommendation statement. Ann Intern Med. 2013;159:210-8.

6. Kuehn BM. Despite benefit, physicians slow to offer brief advice on harmful alcohol use. JAMA 2008;299: 751-3.

7. Cunningham RM, Harrison SR, McKay MP, et al. National survey of emergency department alcohol screening and intervention practices. Ann Emerg Med 2010;55:556-62.

8. Pilowsky DJ, Wu LT. Screening for alcohol and drug use disorders among adults in primary care: A review. Subst Abuse Rehabil 2012:3 25-34.

9. Hettema J, Steele J, Miller WR. Motivational interviewing. Annu Rev Clin Psychol 2005;1:91-111.

10. Smith PC, Schmidt SM, Allensworth-Davies D, Saitz R. Primary care validation of a single-question alcohol screening test. J Gen Intern Med. 2009;24: 783-8.

11. Sullivan LE, Tetrault JM, Braithwaite RS, Turner BJ, Fiellin DA. A meta-analysis of the efficacy of nonphysician brief interventions for unhealthy alcohol use: Implications for the patient-centered medical home. Am J Addict 2011;20:343-56.

12. Weitzman ER, Nelson TF. College student binge drinking and the "prevention paradox": Implications for prevention and harm reduction. J Drug Educ 2004;34:247-65.

13. Rehm J, Gmel G, Sempos CT, Trevisan M. Alcoholrelated morbidity and mortality. Alcohol Res Health 2003;27,39-51.

14. Dawson DA, Goldstein RB, Saha TD, Grant BF. Changes in alcohol consumption: United States, 2001-2002 to 2012-2013. Drug Alcohol Depend 2015;148:56-61.

15. Grant BF, Goldstein RB, Saha TD, et al. The epidemiology of DSM-5 alcohol use disorder: Results from the National Epidemiologic Survey on Alcohol and Related Conditions III. JAMA Psychiatry 2015; 72:757-66.

16. Trust for America's Health. The Facts Hurt, 2015. Available from: http://healthyamericans.org/reports/ injuryprevention $15 /$ release.php? stateid=NM. Accessed June 26, 2016.

17. New Mexico Department of Health. New Mexico Substance Abuse Epidemiology Profile, 2017. Available from: https://nmhealth.org/data/view/substance/ 1862/. Accessed August 10, 2017.

18. Improving Chronic Illness Care. Available from: http://www.improvingchroniccare.org/index.php? p=ACIC_Survey\&s=35. Accessed August 11, 2017.

19. Harris PA, Taylor R, Thielke R, Payne J, Gonzalez N, Conde JG. Research electronic data capture (REDCap)_A metadata-driven methodology and workflow process for providing translational research informatics support. J Biomed Inform 2009; 42:377-81.

20. D'Amico EJ, Paddock SM, Burnam A, Kung FY. Identification of and guidance for problem drinking by general medical providers: Results from a national survey. Med Care 2005;43:229-36.

21. Friedmann PD, McCullough D, Chin MH, Saitz R. Screening and intervention for alcohol problems: A National survey of primary care physicians and psychiatrists. J Gen Intern Med 2000;15:84-91.

22. Nutting PA, Miller WL, Crabtree BF, Jaen CR, Stewart EE, Stange KC. Initial lessons from the First National Demonstration Project on practice transformation to a patient-centered medical home. Ann Fam Med 2009;7:254-260.

23. Williams EC, Johnson ML, Lapham GT, et al. Strategies to implement alcohol screening and brief intervention in primary care settings: A structured literature review. Psychol Addict Behav 2011;25(2):206.

24. Mittman BS. Implementation science in health care. In: Brownson RC, Colditz GA, Proctor EK, eds. Dissemination and implementation research in health: Translating science to practice. New York, NY: Oxford University Press, 2012; 400418.

25. Rogers EM. Diffusion of innovations. 5th ed. New York, NY: Free Press; 2003.

26. Saitz R. 'SBIRT' is the answer? Probably not. Addiction 2015;110:1416-7. 
27. National Institute on Alcohol Abuse and Alcoholism. Drinking Levels Defined. Available from: http:// www.niaaa.nih.gov/alcohol-health/overview-alcoholconsumption/moderate-binge-drinking. Accessed November 9, 2016.

28. Office of Disease Prevention and Health Promotion. Appendix 9: Alcohol. Available from: http://health. gov/dietaryguidelines/2015/guidelines/appendix-9/. Accessed November 9, 2016.

29. Saitz R, Alford DP, Bernstein J, Cheng DM, Samet J, Palfai T. Screening and brief intervention for unhealthy drug use in primary care settings: Random- ized clinical trials are needed. J Addict Med 2010;4: 123-30.

30. Chartier K, Caetano R. Ethnicity and health disparities in alcohol research. Alcohol research \& health: the journal of the National Institute on Alcohol Abuse and Alcoholism 2009;33(1-2):152160 .

31. The InSight Project Research Group. SBIRT outcomes in Houston: Final report on InSight, a hospital district-based program for patients at risk for alcohol or drug use problems. Alcohol Clin Exp Res 2009;33:1374-1381. 